\title{
RECURRENCE AND INCOMPRESSIBILITY
}

\section{CHARLES W. WILLIAMS}

1. Introduction. A classical dynamical system as defined by a system of ordinary differential equations may be considered as a oneparameter transformation group acting on a topological space. Extensive results concerning such systems have been obtained by various authors (see, for example, G. D. Birkhoff [1] ${ }^{1}$ ).

In this paper a generalized dynamical system is considered, such a system being defined as a topological group of transformations acting on a topological space. Basic results for such systems have been obtained by Gottschalk and Hedlund [2], and the fundamental concepts in their paper are adopted here.

The problem of characterizing by an incompressibility property the properties of pointwise periodicity and recurrence in the case of a topological space has been considered by G. T. Whyburn [4]. It would seem of interest to obtain analogous results in the generalized dynamical system. This is the objective of the present paper.

The definitions of periodicity, recurrence, regional recurrence, and stability are stated in the generalized dynamical system so that they reduce to the classical ones in case the group is the group of reals.

2. Definitions. Let $X$ be a separable metric space and let $T$ be a multiplicative abelian topological group with identity element $e$. Let $T$ act as a transformation group on $X$ in the sense that to each pair $(x, t)$. where $x \in X$ and $t \in T$, there is assigned a point of $X$ denoted by $x t$ such that $x e=x,(x t) s=x(t s)$ for $x \in X$ and $t$ and $s$ in $T$, and such that the function $x t$ defined on the topological product $X \times T$ defines a continuous transformation of $X \times T$ into $X$.

In the topological group $T$, we assume that there exists a compact neighborhood $U$ of $e$ which generates $T$, that is, $T=\bigcup_{n=1}^{\infty} U^{n}$. A subset $S \subset T$ is said to be a semigroup provided that $S S \subset S$.

The following definitions were introduced by Gottschalk and Hedlund [2].

(2.1) Definition. A semigroup $S \subset T$ is said to be a replete semigroup provided that $S$ contains some translate of each compact subset of $T$.

(2.2) Definition. A subset $A \subset T$ is said to be an extensive set

Presented to the International Congress of Mathematicians, September 6, 1950 under the title Incompressibility and periodicity; received by the editors May 11, 1950 and, in revised form, November 15, 1950.

${ }^{1}$ Numbers in square brackets refer to the bibliography at the end of the paper. 
provided that $A$ intersects every replete semigroup in $T$.

(2.3) Definition. A subset $D \subset T$ is said to be relatively dense provided that $T=D K$ for some compact subset $K C T$.

3. Periodicity and incompressibility.

(3.1) Definition. A point $x \in X$ is said to be periodic under $T$, provided that there exists a relatively dense subgroup $G \subset T$, such that $x G=x[2]$.

The set of all points of $X$ which are periodic under $T$ will be denoted by $P$.

We note the following equivalent characterization of a periodic point.

(3.2) LEмma. In order that a point $x \in X$ be a periodic point under $T$, it is necessary and sufficient that $x \in x S$ for every replete semigroup $S \subset T$.

The proof of this lemma follows immediately from the fact that $G$ is a relatively dense subgroup provided that $G$ is an extensive subgroup [2, Lemmas 10 and 14].

It is easily proved that the set of periodic points of $X$ is an invariant set.

(3.3) Definition. The transformation group $T$ is said to be pointwise periodic provided that every point of $X$ is periodic under $T$.

The following generalization of a theorem due to G. T. Whyburn [4] has been proved by Gottschalk and Hedlund [2, Theorem 8].

(3.4) THEOREM. In order that $T$ be pointwise periodic, it is necessary and sufficient that if $M$ be a subset of $X$ and $S$ a replete semigroup in $T$ such that $M S \subset M$, then $M-M S=\varnothing$.

The following theorem yields a characterization of a property of the set of periodic points of $X$ in terms of an incompressibility property.

(3.5) TheOREM. In order that the periodic points of $X$ form a residual set in $X$, it is necessary and sufficient that if $M$ is a subset of $X$ and $S$ is a replete semigroup in $T$ such that $M S \subset M$, then $M-M S$ is a set of the first category.

Before proceeding to the proof of the theorem several lemmas will be proved.

(3.6) Lemma. Let $S$ be a replete semigroup in $T$. Let $A$ be the set of all $x \in X$ such that $x \notin x S$. There exists a subset $D \subset X$ such that $A \subset D$ $\cup D S \cup D S^{-1}$, and the three sets $D, D S$, and $D S^{-1}$ are mutually disjoint. 
Proof. With respect to the replete semigroup $S$, we say that the subset $B$ has the property $\mathrm{P}$ provided that $B \cap B S=\varnothing$. It is easily verified that the property $\mathrm{P}$ has finite character. It follows from Zorn's lemma that if $A \neq \varnothing$, there exists a set $D$ containing $x$ which is maximal with respect to property $\mathrm{P}$.

Since $D$ has property $\mathrm{P}, D \cap D S=\varnothing$, and thus also $D \cap D S^{-1}=\varnothing$. Now suppose that $D S \cap D S^{-1} \neq \varnothing$, that is that there exist $d_{1}, d_{2} \in D$, and $s_{1}, s_{2} \in S$, such that $d_{1} s_{1}=d_{2} s_{2}^{-1}$. Then $d_{1} s_{1} s_{2}=d_{2}$ and $d_{2} \notin D$. We conclude that $D, D S$, and $D S^{-1}$ are mutually disjoint.

Suppose that there exists a point $x \in A$ such that $x \in D \cup D S \cup D S^{-1}$. Let $D^{*}=D \cup x$. If $D^{*} \cap D^{*} S \neq \varnothing$, then there exist $d_{1}^{*}, d_{2}^{*} \in D^{*}$ and $s \in S$, such that $d_{1}^{*}=d_{2}^{*} s$. If $d_{1}^{*}, d_{2}^{*} \in D$, then $D \cap D S \neq \varnothing$, which is impossible. If $d_{1}^{*} \in D$ and $d_{2}^{*}=x$, then $d_{1}^{*}=x s$, or $x=d_{1}^{*} s^{-1} \in D S^{-1}$. This again is impossible. If $d_{1}^{*}=x$ and $d_{2}^{*} \in D$, we infer that $x \in D S$, which is impossible. Finally, if $d_{1}^{*}=d_{2}^{*}=x$, that is, that $x=x s$, then $x \notin A$, which is a contradiction. Thus we conclude that $D^{*}$ has property $\mathrm{P}$, and $D$ is not maximal with respect to property $\mathrm{P}$. We conclude that $A \subset D \cup D S \cup D S^{-1}$. If $A=\varnothing$ we define $D=\varnothing$. This completes the proof of the lemma.

A set $D$, the existence of which is assured by the lemma, will be denoted by $D(S)$.

(3.7) LemMA. If $M$ is a subset of $X$ and $S$ is a replete semigroup in $T$ such that $M S \subset M$ implies that $M-M S$ is a set of the first category, then if $D$ is a set $D(S)$, it follows that $D$ is a set of the first category.

Proof. Let $E=D \cup D S$. Then $E S=D S \subset E$, and by hypothesis $E-E S$ is a set of the first category. Since $D \cap D S=\varnothing$, we infer that $D \subset E-E S$, thus $D$ is also of the first category, and the proof of the lemma is completed.

(3.8) Lemma. Let $S$ be a replete semigroup in $T$ and let $D$ be a set $D(S)$. Then if for every subset $M$ of $X$ and replete semigroup $S^{*}$ in $T$ it is true that $M-M S^{*}$ is a set of the first category when $M S^{*} \subset M$, it follows that the sets $D S$ and $D S^{-1}$ are of the first category.

Proof. Let $V$ be a symmetric compact neighborhood of $e \in T$. Define $S^{*}=\bigcap_{8} \in v^{2} S v$. Then $S^{*}$ is a replete semigroup in $T$ [2, Lemma 4]. Define $Q=D V$. Suppose that $Q \cap Q S^{*} \neq \varnothing$. Then there exist $d_{1}, d_{2} \in D$, $v_{1}, v_{2} \in V$, and $s^{*} \in S^{*}$ such that $d_{1} v_{1}=d_{2} v_{2} s^{*}$ or $d_{1}=d_{2} v_{2} v_{1}^{-1} s^{*}$. However, $v_{2}^{-1} v_{1} \in V^{2}$, and we infer that $s^{*} \in S v_{2}^{-1} v_{1}$, or $d_{1}=d_{2} s$, where $s \in S$. This is impossible since $D \cap D S=\varnothing$, and thus we conclude that $Q \cap Q S^{*}$ $=\varnothing$.

Let $R=Q \cup Q S^{*}$. Surely $R S^{*}=Q S^{*} \subset R$, and since $Q \cap Q S^{*}=\varnothing$ it 
follows that $Q \subset R-R S^{*}$. Since $R-R S^{*}$ is a set of the first category, we infer that $Q$ is likewise of the first category.

There exists a countable sequence $s_{1}, s_{2}, \ldots$ in $S$ such that $S \subset \bigcup_{i=1}^{\infty} V s_{i}$. Since $D S=D\left(\bigcup_{i=1}^{\infty} V s_{i}\right) \subset \bigcup_{i=1}^{\infty} D V s_{i}=\bigcup_{i=1}^{\infty} Q s_{i}$ and $Q$ is a set of the first category, each of the sets $Q s_{i}$ is of the first category for $i=1,2, \cdots$, we infer that $D S$ is also of the first category. By a similar argument it follows that $D S^{-1}$ is of the first category, and the proof of the lemma is completed.

(3.9) Corollary. Let $S$ be a replete semigroup in $T$ and let $A$ be the set of all $x \in X$ such that $x \notin x S$. If for every subset $M$ of $X$ and replete semigroup $S^{*}$ in $T$ satisfying $M S^{*} \subset M$ it is true that $M-M S^{*}$ is a set of the first category in $X$, then the set $A$ is of the first category.

Proof. The proof follows from Lemmas 3.6, 3.7, and 3.8.

We now proceed with the proof of Theorem 3.5.

Proof. We first establish the necessity of the condition. Let $M$ be a subset of $X$ and $S$ a replete semigroup in $T$ such that $M S \subset M$. If $x \in M$ and $x$ is a periodic point. in $X$, then $x \in x S \subset M S$. It follows that $M-M S \subset X-P$, where $P$ denotes the set of periodic points of $X$, and we conclude that $M-M S$ is of the first category which establishes the necessity of the condition.

If the group $T$ is compact, the single element $e \in T$ is itself a relatively dense subgroup in $T$, and it follows that each point of $X$ is a periodic point.

If $T$ is not a compact group, corresponding to a given symmetric compact neighborhood $V$ of $e$, there exists a basis $S_{1}, S_{2}, \ldots$ for the replete semigroups of $T$, such that $S_{i} \cap V=\varnothing, i=1,2, \cdots$, and such that for any replete semigroup $S$, there exists an integer $n$ such that $S_{n} \subset S[2$, Lemmas 9 and 10].

For each positive integer $i$, let $A_{i}$ be the set of all $x \in X$ such that $x \notin x S_{i}$. We show that $X-P \subset \bigcup_{i=1}^{\infty} A_{i}$. For suppose that $x \in X-P$. Then there exists a replete semigroup $S \subset T$, such that $x \notin x S$. There exists a positive integer $i$, such that $S_{i} \subset S$, and therefore $x \notin x S_{i}$, or $x \in A_{i}$.

But from Corollary 3.9, each $A_{i}$ is a set of the first category, $i=1,2, \cdots$, and we infer that $X-P$ is a set of the first category. We conclude that $P$ is residual in $X$ and the condition is sufficient.

\section{Recurrence and incompressibility.}

(4.1) Definition (cf. [2]). A point $x \in X$ is said to be recurrent under $T$ provided that to each neighborhood $U$ of $x$ there corresponds an extensive set $A \subset T$ such that $x A \subset U$. 
The set of all points of $X$ which are recurrent under $T$ we denote by $R$. We note also the following equivalent characterization of a recurrent point.

We adopt the following convention throughout the remainder of the paper. For $Y \subset X$ and $H \subset T,[Y H]$ denotes the closure of the set $Y H$.

(4.2) Lemma. In order that a point $x \in X$ be recurrent under $T$ it is necessary and sufficient that $x \in[x S]$ for every replete semigroup $S \subset T$.

It is easily proved that the set of recurrent points of $X$ is an invariant set.

(4.3) Definition. The transformation group $T$ is said to be pointwise recurrent provided that each point of $X$ is recurrent under $T$.

The following theorem [2, Theorem 7] states a characterization of pointwise recurrence in terms of an incompressibility property.

(4.4) THEOREM. In order that $T$ be pointwise recurrent it is necessary and sufficient that if $M$ be a closed subset of $X$ and $S$ a replete semigroup in $T$ such that $M S \subset M$, then $M S=M$.

In general, the transformation group is not pointwise recurrent. However, it is still possible to characterize the set $R$ in terms of an incompressibility property.

(4.5) Lemma. If $M$ is a closed subset of $X$ and $S$ is a replete semigroup in $T$ such that $M S \subset M$, then there exists a replete semigroup $S^{*} \subset S$, such that for all $x \in M-M S, x \notin\left[x S^{*}\right]$.

Proof. Let $K$ be the closure of a compact neighborhood of $e$ which generates $T$. Since $S$ is a replete semigroup in $T$ there exists an element $t \in T$ such that $K t \subset S$. Define $H=K t$. $H$ is then also a compact set and $S^{*}=\bigcup_{n=1}^{\infty} H^{n}$ is a replete semigroup which is contained in $S$. Since $H \subset S$, and by hypothesis $M S \subset M$, we infer that $M H \subset M S \subset M$, and hence by induction that $M H^{n} \subset M H$ for $n=1,2, \cdots$. Thus $M S^{*} \subset M H$. Now suppose that $x \in\left[x S^{*}\right]$, and that $x \in M$. Then $x \in\left[x S^{*}\right] \subset\left[M S^{*}\right] \subset[M H]=M H \subset M S$. Thus for all $x \in M-M S$, $x \in\left[x S^{*}\right]$. From this we also conclude that $x \in X-R$.

(4.6) Definition (cf. [2]). The transformation group $T$ is said to be regionally recurrent provided that to each open set $U \subset X$ there corresponds an extensive set $A \subset T$ such that for each $a \in A, U \cap U a$ $\neq \varnothing$.

The following theorem states the desired incompressibility property which characterizes a property of the set $R$.

(4.7) Theorem. If $X$ is a complete metric space, in order that the 
recurrent points of $X$ form a residual set in $X$, it is necessary and sufficient that if $M$ is a closed subset of $X$ and $S$ a replete semigroup in $T$ such that $M S \subset M$, then $M-M S$ is a set of the first category.

Proof. The necessity of the condition follows directly from Lemma 4.5 , for $M-M S \subset X-R$ which is a set of the first category by hypothesis.

In order to show the sufficiency of the condition, it is enough to show that $T$ is regionally recurrent [2, Theorem 3$]$. If this were not the situation, there would exist an open set $U \subset X$ and a replete semigroup $S \subset T$, such that $U \cap U S=\varnothing$. Now let $Z$ be the closure of a sphere of positive radius which is contained wholly in $U$. Surely $U \cap Z S=\varnothing$, from which we infer that $U \cap[Z S]=\varnothing$, and also that $Z \cap[Z S]=\varnothing$.

We define $M=Z \cup[Z S]$, which is a closed set. Certainly $M S \subset M$, and thus by hypothesis $M-M S$ is a set of the first category. But since $Z \subset M-M S$, we infer that $Z$ is also of the first category. This, however, is impossible in a complete metric space, and we infer that $T$ must be regionally recurrent. Finally then $R$ is a residual set, and the proof of the theorem is completed.

(4.8) Definition. A point $x \in X$ is said to be wandering under $T$ provided that there exists a neighborhood $U$ of $x$ and a replete semigroup $S \subset T$, such that $U \cap U S=\varnothing$.

It is easy to see that the definition stated here reduces to the corresponding classical one if the group $T$ is the real axis. We denote the set of all wandering points of $X$ by $W$.

(4.9) Definition. The transformation group $T$ is said to be pointwise nonwandering provided that each point of $X$ is not a wandering point under $T$.

Clearly the properties that $T$ be regional recurrent and that $T$ be pointwise nonwandering are equivalent.

We shall now show that if a properly chosen set of the first category is excluded, the space $X$ can be decomposed into two invariant sets such that every point of the first is a recurrent point and every point of the second is a wandering point.

(4.10) Theorem. Let $X$ be a complete metric space. The set $R \cup W$ is a residual set in $X$.

Proof. Since both $R$ and $W$ are invariant sets, it follows readily that $\bar{R} \cup \bar{W}$ is a closed invariant set. The set $Y=X-(\bar{R} \cup \bar{W})$ is then an open invariant set.

It is easily proved that no point of $Y$ is a wandering point in the 
set $Y$ and no point of $\bar{Y}$ is a wandering point in the set $\bar{Y}$.

Define $K=\bar{Y}$, and let $R_{K}$ denote the set of all points of $K$ which are recurrent in $K$. Since every point of $K$ is nonwandering in $K, T$ is regionally recurrent in $K$, and therefore $R_{K}$ is dense in $K[2$, Theorem 3, Corollary 2].

Certainly $R_{K} \subset R$, and therefore $Y \subset K=\bar{R}_{K} \subset \bar{R}$. But $Y \subset X-\bar{R}$, and we conclude that $Y=\varnothing$. Thus we infer that $X=\bar{R} \cup \bar{W}$.

The set $\bar{W}-W$ is nowhere dense in $X$ and since $R$ is a residual set in $\bar{R}, \bar{R}-R$ is a set of the first category in $\bar{R}$ and hence also in the space $X$. Finally then $X-(R \cup W)=(\bar{R}-R) \cup(\bar{W}-W)$ is a set of the first category and we infer that $R \cup W$ is a residual set in $X$. This completes the proof of the theorem.

5. Stability and incompressibility.

(5.1) Definition. If $S$ is a replete semigroup in $T$, the set $A \subset S$ is said to be extensive in $S$ provided that $A \cap S^{*} \neq \varnothing$ for every replete semigroup $S^{*}$ of $T$ which is contained in $S$.

(5.2) Definition. If $S$ is a replete semigroup in $T$, a point $x \in X$ is said to be recurrent under $S$ provided that corresponding to every neighborhood $U$ of $x$, there exists a set $A$, extensive in $S$, such that $x A \subset U$.

We denote the set of all points of $X$ which are recurrent under $S$ by $R(S)$.

(5.3) Definition. The point $x \in X$ is said to be stable with respect to $S$, a replete semigroup in $T$, provided that $[x S]$ is a compact set.

We denote the set of all $x \in X$ which are stable with respect to $S$ by $\Sigma(S)$, and the complementary set $X-\Sigma(S)$ by $U(S)$, that is, the set of points of $X$ which are unstable with respect to $S$.

(5.4) Lemma. If $M$ is a compact subset of $X$ and $S^{*}$ is a replete semigroup in $T$ such that $M S^{*} \subset M$, then for all $x \in M-M S^{*}, x \notin U\left(S^{*}\right)$ $\cup R\left(S^{*}\right)$.

Proof. By Lemma 4.5 there exists a replete semigroup $S^{\prime} C S^{*}$, such that for all $x \in M-M S^{*}, x \in\left[x S^{\prime}\right]$. Thus we infer that $x \in R\left(S^{*}\right)$. Also $x S^{*} \subset M S^{*} \subset M$. Therefore $\left[x S^{*}\right]$ is a compact set so that $x \notin U\left(S^{*}\right)$. This completes the proof of the lemma.

(5.5) THEOREM. In order that $X=U(S) \cup R(S)$ for every replete semigroup $S \subset T$, it is necessary and sufficient that if $M$ is a compact subset in $X$ and $S^{*}$ is a replete semigroup in $T$ such that $M S^{*} \subset M$, then $M-M S^{*}=\varnothing$.

Proof. The necessity of the condition follows directly from Lemma 5.4. 
Now suppose that the condition is satisfied and that there exists a point $x \notin U(S) \cup R(S)$ for some replete semigroup $S \subset T$. In particular $x \notin R(S)$, so that there exists a replete semigroup $S^{*} \subset S$ such that $x \notin\left[x S^{*}\right]$. Let $M=x \cup\left[x S^{*}\right]$. Then $x \in M-M S^{*}$. Since $x \notin U(S)$, $[x S]$ is a compact set, and since $M=x \cup\left[x S^{*}\right] \subset x \cup[x S]$ it follows that $M$ is also a compact set. We have $M S^{*} \subset\left[x S^{*}\right] \subset M$, and therefore by hypothesis $M-M S^{*}=\varnothing$ which contradicts the conclusion that $x \in M-M S^{*}$. We infer that $X=U(S) \cup R(S)$, which completes the proof of the sufficiency of the condition.

(5.6) Definition. The point $x \in X$ is said to be stable provided that $[x T]$ is a compact set.

The set of all $x \in X$ which are not stable will be denoted by $U$.

(5.7) Definition. The point $x \in X$ is said to be diffuse provided that $x \in U(S)$ for every replete semigroup $S \subset T$.

The set of all $x \in X$ which are diffuse will be denoted by $D$.

(5.8) Corollary. If $X=R \cup D$, and if $M$ is a compact subset in $X$ and $S$ is a replete semigroup in $T$ such that $M S \subset M$, then $M-M S=\varnothing$.

Proof. For every replete semigroup $S \subset T, R \subset R(S)$ and $D \subset U(S)$. Thus $X=R \cup D \subset R(S) \cup U(S) \subset X$, and therefore $X=R(S) \cup U(S)$. The corollary now follows from Theorem 5.5.

(5.9) CoRollary. If for every compact set $M \subset X$ and for every replete semigroup $S \subset T$ for which $M S \subset M, M-M S=\varnothing$, then $X$ $=R \cup U$.

Proof. From Theorem 5.5, $X=R(S) \cup U(S)$ for every replete semigroup $S \subset T$. In particular the group $T$ itself is such a replete semigroup and we infer that $X=R(T) \cup U(T)=R \cup U$.

(5.10) THEOREM. If the set $R \cup D$ is a residual set in $X$, and if $M$ is a compact subset of $X$ and $S$ is a replete semigroup in $T$ such that $M S \subset M$, then $M-M S$ is a set of the first category.

Proof. From Lemma 5.4, $M-M S \subset \Sigma(S) \cap[X-R(S)] \subset(X-D)$ $\cap(X-R)$. This last set however is of the first category by hypothesis. Thus we infer that $M-M S$ is of the first category, which completes the proof of the theorem.

(5.11) Theorem. Let $X$ be a locally compact, complete, separable metric space. If, for every compact subset $M \subset X$ and for every replete semigroup $S \subset T$ for which $M S \subset M, M-M S$ is a set of the first category, then the set $R \cup U$ is a residual set in $X$.

Proof. Since the space $X$ is locally compact and separable, there exists a monotone sequence of compact subsets of $X: X_{1} \subset X_{2} \subset \cdots$ 
with the property that if $M$ is a compact subset of $X, M \subset X_{n}$ for some positive integer $n$. Let $S_{1}, S_{2}, \cdots$ be a countable basis for the replete semigroups in $T$.

For positive integers $m$ and $n$, we say that the point $x \in X$ is in the set $E(n, m)$ provided that the distance $d\left(x,\left[x S_{n}\right]\right) \geqq 1 / m$. The set $E(n, m)$ is then a closed set.

For positive integers $m$ and $n$, let $G(n, m)$ denote the set of all $x \in X$ such that $x \in E(n, m)$ and $x S_{n} \subset X_{m}$. Then $G(n, m)$ is also a closed set.

We show that $(X-U) \cap(X-R) \subset \cup_{n, m=1}^{\infty} G(n, m)$. Let $x \in(X-R)$ $\cap(X-U)$. Since $x \notin R$, there exists a replete semigroup $S \subset T$ such that $x \notin[x S]$. There exists a positive integer $n$ such that $S_{n} \subset S$, and we infer that $x \in\left[x S_{n}\right]$. There exists a positive integer $q$, such that for all integers $i \geqq q, d\left(x,\left[x S_{n}\right]\right) \geqq 1 / i$. Thus we conclude that $x$ $\in E(n, i)$ for all $i \geqq q$. Now since $x \in X-U,[x T]$ is a compact set and therefore $\left[x S_{n}\right]$ is compact. Hence there exists a positive integer $k$ such that $\left[x S_{n}\right] \subset X_{k}$, and thus for all positive integers $p \geqq k$, $\left[x S_{n}\right] \subset X_{p}$. Let us now choose an integer $m$ such that $m>p+k$. We then have $x \in E(n, m)$ and $\left[x S_{n}\right] \subset X_{m}$, from which we infer that $x \in G(n, m)$. Thus $(X-U) \cap(X-R) \subset \cup_{n, m=1}^{\infty} G(n, m)$.

Suppose that the set $G(n, m)$ is not nowhere dense. There exists an open set $V \subset G(n, m)$ of diameter less than $1 / m$ and such that $\bar{V}$ is a compact set. Let $M=\bar{V} \cup\left[V S_{n}\right]$. Since $V S_{n} \subset X_{m},\left[V S_{n}\right]$ is a compact set and therefore $M$ is also compact. But $M S_{n} \subset M$, so that, by hypothesis, $M-M S_{n}$ is a set of the first category. Since $V$ $C E(n, m)$, and since the diameter of $V$ is less than $1 / m$, we infer that $V \cap\left[V S_{n}\right]=\varnothing$. But $M S_{n} \subset\left[V S_{n}\right]$, and therefore $V \subset M-M S_{n}$, which is impossible in a complete metric space. Thus $G(n, m)$ is a nowhere dense set and we conclude that $(X-U) \cap(X-R)$ is a set of the first category. Therefore $U \cup R$ is a residual set in $X$ and the proof of the theorem is completed.

\section{BIBLIOGRAPHY}

1. G. D. Birkhoff, Dynamical systems, Amer. Math. Soc. Colloquium Publications, vol. 9, New York, 1927.

2. W. H. Gottschalk, and G. A. Hedlund, The dynamics of transformation groups Trans. Amer. Math. Soc. vol. 65 (1949) pp. 348-359. 1946.

3. L. Pontrjagin, Topological groups, Princeton Mathematical Series, Princeton,

4. G. T. Whyburn, Analytic topology, Amer. Math. Soc. Colloquium Publications, vol. 28, New York, 1942.

UNIVERSITY OF VIRGINIA AND

WASHINGTON AND LEE UNIVERSITY 